\title{
Noch keine Chancengleichheit im Gesundheitssystem
}

\section{Integration bedarf einer migrationssensiblen Präven- tionspolitik}

Im Sommer letzten Jahres lud die Bundeskanzlerin Migranten und Vertreter aller politischen Ebenen und gesellschaftlichen Gruppen zum Integrationsgipfel ein. Gedacht war der Gipfel als Auftakt zu einem fortlaufenden Dialog, an dessen Abschluss ein Nationaler Integrationsplan stehen soll. Im Vorfeld legte das Bundeskabinett ein Positionspapier zur Politik der Integrationsbeauftragten vor, die die Integration von zugewanderten Menschen als politische Schlüsselaufgabe identifiziert. Danach verfolgt Integrationspolitik das Ziel einer möglichst guten und breiten Verwirklichung von Beteiligungsmöglichkeiten in allen Lebensbereichen - explizit auch im Gesundheitsbereich.

\section{Michael Bellwinkel}

Immerhin 19 Prozent der Bevölkerung im Zuwanderungsland Deutschland haben heute einen Migrationshintergrund, im Jahr 2010 könnten nach aktuellen Prognosen 40 Prozent der Unter-40-Jährigen eine Zuwanderungsgeschichte aufweisen. Wie steht es aber um bisherige Integrationsbemühungen und -erfolge des Lebensbereichs Gesundheit und seiner Versorgungssysteme?

Tatsache ist, dass informationsbedingte, kulturelle und kommunikative Barrieren die effektive Nutzung des Gesundheitswesens behindern und dadurch auf der einen Seite erhebliches Leid, auf der anderen Seite erheblichen Aufwand und Kosten verursachen. Ein Beispiel aus der Praxis: Ein älterer Mann aus der Türkei mit schlechten Deutschkenntnissen berichtet seinem Arzt von Herzschmerzen. Dieser veranlasst daraufhin zahlreiche kardiologische Untersuchungen einschließlich stationären Aufenthalts in einer Fachklinik - ohne Ergebnisse. Erst ein hinzugezogener türkischer Landsmann erklärt, dass im türkischen Kulturkreis Herzschmerz Heimweh bedeutet. Das Verständnis solcher interkultureller Unterschiede könnte Beratungsleistungen und medizinische Versorgung treffsicherer, weniger fehleranfällig und viel effizienter für alle Beteiligten gestalten.

Michael Bellwinkel, BKK Bundesverband, Leiter des Referats "Gesundheitsförderung und Selbsthilfe"
Grafik 1: Migrationshintergrund der Bevölkerung in Deutschland 2005

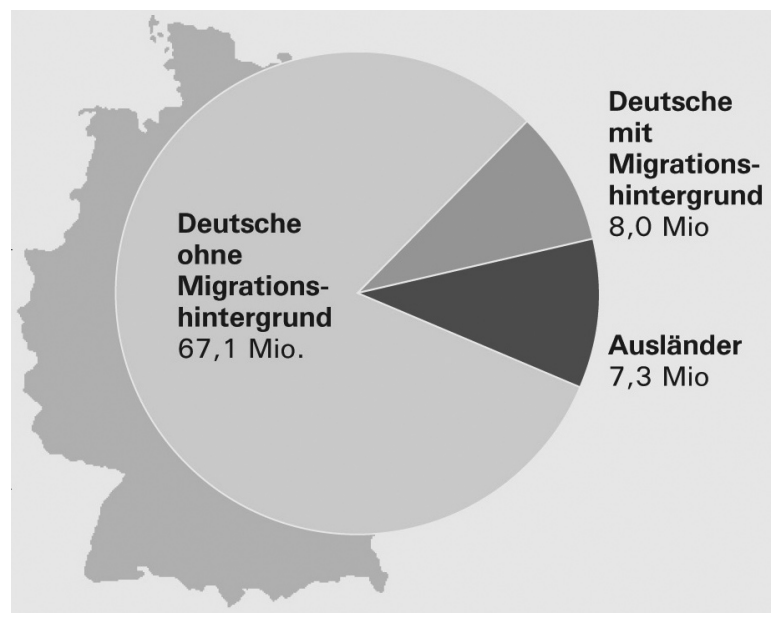

Quelle: Mikrozensus; Grafik: BKK Bundesverband

Das Beispiel und der aktuelle Diskurs um Integration rücken die Frage nach der gegenwärtigen praktischen Leistungseffektivität der gesundheitlichen Versorgungssysteme in Bezug auf Menschen mit Migrationshintergrund näher ins Blickfeld. Aufgrund der Wechseldynamik zwi- 


\section{THEMA}

schen Integration und Gesundheit kann die gesundheitliche Lage Integrationsverläufe mit allen ökonomischen, sozialen, kulturellen und politischen Implikationen abbilden. Das heißt, dass die soziale und politische Integration von Migranten und die Verbesserung von Strukturen, die einen guten Gesundheitszustand fördern, sich wechselseitig bedingen. Umgekehrt spielt das Gesundheitssystem selbst eine nicht unwesentliche Rolle bei der Integration von Migranten.

\section{Gesundheitliche Faktoren bei Migranten}

Festzuhalten ist vor allem zunächst, dass Migration als solche nicht krank macht oder ein generelles Gesundheitsrisiko darstellt. Vielmehr birgt Migration gleichermaßen Chancen wie spezifische Risiken für die Gesundheit. Sie bietet die Möglichkeit zur Neuorientierung und zur Nutzung alternativer ökonomischer und sozialer Ressourcen. Als gesundheitsfördernde Ressource gilt zum Beispiel der erfolgreiche Umgang mit Risiken und Belastungen. Durch Migrationserfahrungen erlernte Bewältigungsstrategien können den Einzelnen unter bestimmten Voraussetzungen flexibler und belastungsfähiger werden lassen. Hinzu kommt, dass in einer von Globalisierung erfassten und somit zunehmend an Diversity-Konzepten interessierten Wanderungswelt Fremd-Sein an sich eine wertvolle Ressource sein kann: mindestens zwei Sprachen verstehen, sich in mehreren Kulturen zurechtfinden oder eine Vermittlerrolle einnehmen. Leider wird dies auf beiden Seiten noch zu wenig in positiver Weise wahrgenommen und genutzt. Insbesondere im Gesundheits- und Sozialsystem gilt es in diesem Punkt zu erkennen, was in der Wirtschaft mit Blick auf den ökonomischen Nutzen als „Standortvorteil durch Diversity“ längst Allgemeingut ist.

\section{Grafik 2: Übergewicht und Adipositas bei Schulanfängern nach Migrationshintergrund}

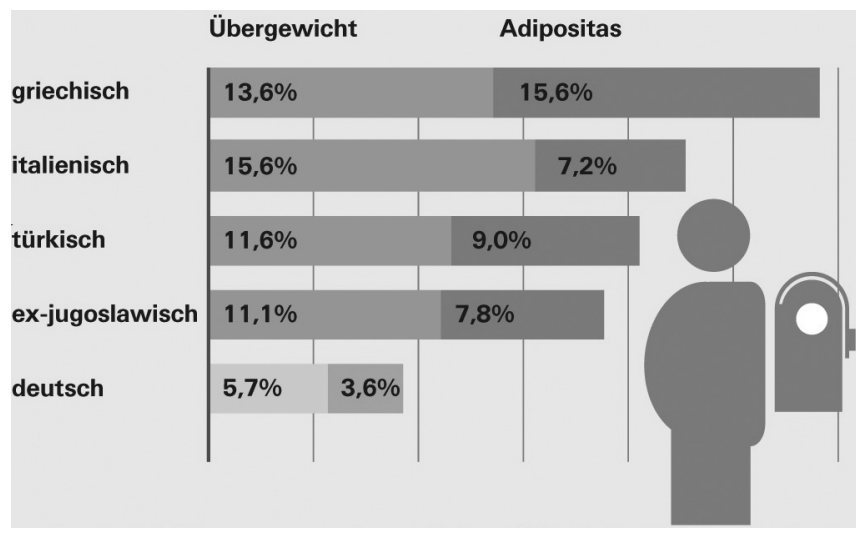

Quelle: Schuleingangsuntersuchung München 2003;

Grafik: BKK Bundesverband

Gelingt nach erfolgreicher Migration der Prozess der Integration nicht oder nur unzureichend, kann Migration als belastend, verunsichernd und somit auch krankmachend erlebt werden. Vor allem mit der Migration verbundene traumatische Erfahrungen können eine große gesundheitliche Belastung darstellen. Es ist davon auszugehen, dass die Umstände der Migration sowie ethnomedizinische Hintergründe einen Einfluss auf die gesundheitliche Situation haben. Eine Verengung des Blicks nur auf die kulturelle Prägung durch das Herkunftsland ist aber nicht zielführend. Viel wichtiger und von gesundheitsrelevanter Bedeutung sind der sozioökonomische Status, persönliche und kollektive biografische Erfahrungen sowie alters- und geschlechtsspezifische Faktoren nach der Migration im Zuwanderungsland.

Ebenfalls festzuhalten ist, dass Migranten nicht selten in mehrfacher Hinsicht von sozialer Ungleichheit von Gesundheitschancen betroffen sind. Auch hier muss eindringlich vor einer Pauschalisierung gewarnt werden: Den oder die sozial benachteiligten Migranten gibt es nicht. Belegbar ist jedoch, dass eine von Migration geprägte Lebenssituation immer noch eher mit einem niedrigen sozioökonomischen Status einhergeht: geringes Einkommen, überproportionale Betroffenheit von Arbeitslosigkeit, mangelnde soziale Integration, geringere Bildungschancen etc.. Das biografische Ereignis Migration geht demgemäß oft einher mit sozialer Benachteiligung im Aufnahmeland, was wiederum Auswirkungen auf die Gesundheit hat.

Einige Grundannahmen spielen beim Zugang zum Thema „Gesundheitliche Lage von Migranten“ eine wesentliche Rolle. Zum einen teilen Migranten die gesundheitlichen Probleme einheimischer Patienten, wenn sie sich in ihrem sozialen Status ähneln. Und zum anderen korrelieren die Unterschiede im Gesund- und Kranksein mit Variablen der Sozialisation und Herkunft. Diese Aussagen finden ihre empirische Bestätigung in den Auswertungen BKK-Systems der Arbeitsunfähigkeitstage (AU-Tage) pro Jahr. So weisen Arbeitnehmer mit türkischem Migrationshintergrund mit 18 AU-Tagen doppelt so viele AU-Tage auf wie Migranten aus Asien. Während türkische und türkischstämmige Migranten überwiegend in körperlich belastenden Berufen tätig sind, üben asiatische Migranten häufiger hoch qualifizierte Dienstleistungstätigkeiten aus. Neben diesen Faktoren der Art der ausgeübten Berufstätigkeit sind vor allem jene von Bedeutung, die danach fragen, inwieweit Migranten eine adäquate kurative und präventive Versorgung erhalten und selbst in Anspruch nehmen. Die Datenlage der Gesetzlichen Krankenversicherung (GKV) zur Integration von Migranten in das präventive Regelangebot ist leider noch meist defizitär.

Präventionsangebote sind in vielen Fällen vornehmlich für einen bestimmten Kulturkreis und am sozialen Mittelstand orientiert konzipiert. Gesundheit ist vorrangig ein Thema der gut situierten, gebildeten Bevölkerungsschicht. Gesundheit als erstrebenswertes Gut, gar als „Life-StyleKonzept“, wird jedoch erst dann handlungsleitend, wenn die Grundbedürfnisse nach Essen, Wohnung, Sicherheit erfüllt sind. Beispiel Früherkennungsleistungen: Trotz grundsätzlicher Zugangsoffenheit zu GKV-Früherkennungsleistungen für alle Menschen außer Asylbewerber sind Migranten hier sowie bei der zahnmedizi- 
Grafik 3: Arbeitsunfähigkeit der Arbeitlosen nach Nationalität

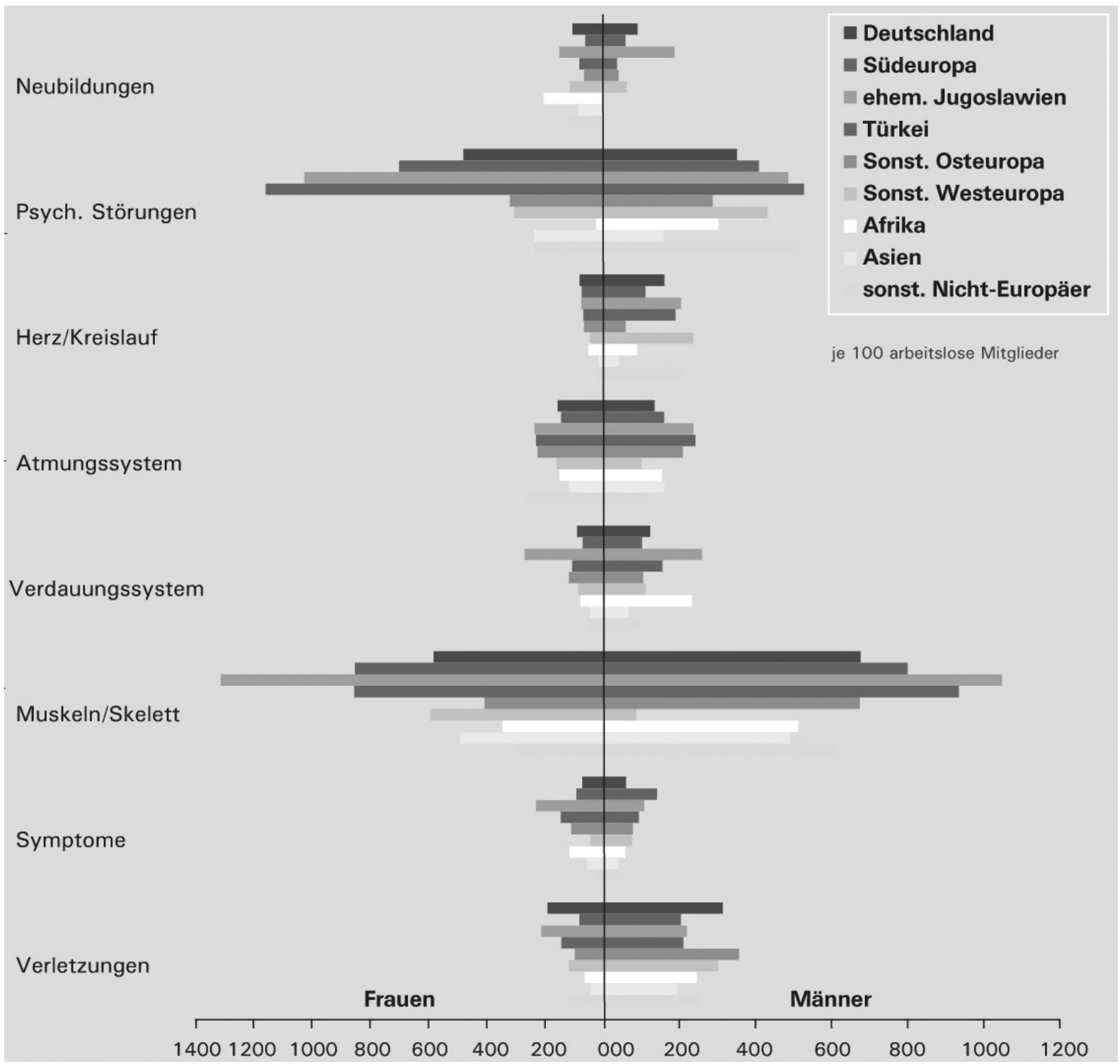

Quelle: BKK Gesundheitsreport 2007

nischen Prophylaxe oder Früherkennungsuntersuchungen in der Schwangerschaft deutlich unterrepräsentiert. Ein Blick auf die HIV-Prävention veranschaulicht besonders deutlich, dass Angebotsformen nicht immer die Lebensrealität von Migranten treffen. Zwar liegt der Anteil der an AIDS erkrankten Migranten nur geringfügig über dem der deutschen Bevölkerung; das Spektrum der Übertragungswege und die Geschlechterstruktur weichen jedoch deutlich von der Mehrheitsbevölkerung ab. In der Regel bestehen andere Voraussetzungen für die HIV-Übertragung durch mögliche differierende kulturelle Sexualitätskonzepte, Rollenmuster und Sexualpraktiken. Die auf die Mehrheitsbevölkerung zugeschnittene Sexualaufklärung und die HIV-Prävention können für diese Zielgruppe daher nur begrenzt wirksam sein und müssten angepasst werden.

\section{Empfehlungen für eine migrationssensible Präventionspolitik}

Gesundheitsförderung für Menschen mit Migrationshintergrund sollte neben übergeordneten Ansätzen in Politik und Lebensraumgestaltung die Ziele interkulturelle Öffnung, ganzheitliche Ressourcenförderung zur Selbstbestimmung und die Prävention spezifischer Risikofaktoren spezieller Risikogruppen beinhalten. Ein Schlüssel zum Erfolg von Maßnahmen zur Gesundheitsförderung von Migrantengruppen ist die Beteiligung der Betroffenen. Die problemassoziierte Zielgruppe ist immer auch Teil der Lösung der mit ihr in Zusammenhang gebrachten Probleme.

Die gesetzlichen Krankenkassen können im Alleingang keine grundsätzlichen Gesellschaftsprobleme kompensie- 
ren oder brachliegende Ressourcen mobilisieren. Jedoch können sie im Rahmen ihres gesetzlich vorgegebenen Spielraums durch ihr Handeln Anstöße liefern und auch den politischen Diskurs befördern. Um bei Migranten primärpräventiv effektiv tätig zu werden, kann beispielsweise die Einbindung in ein Gesamtprogramm zur Umsetzung der präventiven Vorgaben nach Paragraf 20 Abs. 1 SGB V ein wichtiger Faktor sein.

Migrationsspezifische Ansätze, die auf die Beseitigung offensichtlicher Defizite in Prävention, Kuration und Rehabilitation abzielen, werden um so erfolgreicher sein, je mehr sie Teil aktueller „Trends“ werden und nicht als Extralösung in der Schublade „wünschenswert, aber unbezahlbar“ abgelegt werden kann. Der BKK Bundesverband folgt dieser von Professor Dr. Norbert Schmacke erhobenen Forderung. Im Rahmen der soziallagenbezogenen BKK Präventionsinitiative „Mehr Gesundheit für alle“ zielt er auf verschiedene sozial benachteiligte Gruppen, u. a. auch auf benachteiligte Migranten. Mit Projekten zur Gesundheitsförderung mit und für Migranten leistet er einen Beitrag zur Förderung interkultureller Kompetenz in Prävention und Gesundheitsförderung. Die hieraus gewonnenen Erfahrungen sollen den Versicherten mit Migrationshintergrund im BKK System zu einer effektiveren und effizienteren Nutzung ihrer Gesundheitspotenziale und des Gesundheitssystems verhelfen. Gute Beispiele sind etwa „MiMi - Mit Migranten für Migranten“, „Gesundheitsthemen in Sprachkursen“, „Gesund essen mit Freude - kultursensible Koch- und Ernährungskurse“ oder „Dr. Schnupper rät“, die mit Partnern wie dem Ethno-Medizinischen Zentrum Hannover oder der Arbeiterwohlfahrt entwickelt und erprobt werden.

Man kann man es nicht oft genug betonen: Interesse an erfolgreicher Integration haben nicht nur Zugewanderte. Interesse daran muss auch die aufnehmende Bevölkerung haben. Denn eine effektive und effiziente gesundheitliche Versorgung ist nicht nur von größter Bedeutung für die Menschen mit Migrationshintergrund, sondern von zunehmender Bedeutung gerade auch für die versorgenden Fachkräfte und nicht zuletzt für die Kostenträger. An der Gruppe der Migranten wird sichtbar, was sich bezüglich der Mechanismen und Auswirkungen auch auf andere gesellschaftliche Gruppen übertragen lässt. Die verbreitete Annahme der Chancengleichheit zur Nutzung von gesundheitlichen Präventions- und Versorgungsangeboten ist falsch. Vielmehr verhindert sie die Analyse und Verhältnisänderung und zementiert die Chancenungleichheit. Aufbauend auf diesen Erkenntnissen sollte ein übergeordnetes Ziel migrationssensibler Präventionsarbeit entwickelt werden, mit dem vorhandene Potenziale gefördert und Problemstrukturen reduziert werden können. Erfolgreich erprobte Beispiele der Krankenkassen, wie der Betriebskrankenkassen, können den Weg zu einer interkulturellen Öffnung des Gesundheitswesens weisen.

Weitere Informationen: www.bkk.de/mehr-gesundheitfuer-alle und www.bkk-promig.de

\section{Literaturverzeichnis}

- Arbeiterwohlfahrt Bundesverband: Sprechstunde bei Dr. Schnupper. Bilderbuch, Bonn 2005

- Arbeiterwohlfahrt Bundesverband: Dr. Schnupper rät: So bleibt ihr Kind gesund. Faltblatt/Plakate, Bonn 2005

- Bauch: Das gegliederte System der gesundheitlichen Versorgung in Deutschland und Probleme der Migration. In: Türkisch Deutsche Gesundheitsstiftung (Hrsg.): Wissenschaftliche Arbeiten über Gesundheit und Migration, Gießen, 2002

- Beauftragte der Bundesregierung für Migration, Flüchtlinge und Integration (Hrsg.): Gesunde Integration, Dokumentation der Fachtagung für die Ausländerbeauftragten in Deutschland am 20./21.02.2003, Berlin 2003

- Beauftragte der Bundesregierung für Ausländerfragen (Hrsg.): Bericht der Beauftragten der Bundesregierung für Ausländerfragen über die Lage der Ausländer in der Bundesrepublik Deutschland, Berlin/Bonn 2002

- BKK Bundesverband (Hrsg.): Gesundheit Hand in Hand. Das deutsche Gesundheitssystem. Ein Wegweiser für Migrantinnen und Migranten, Essen 2005

- $\quad$ BKK Bundesverband (Hrsg.): Mehr Gesundheit für alle. Zwischenbilanz 2007. Sonderbeilage in: Die BKK, Heft 12/2007

- BKK Bundesverband (Hrsg.): Das ABC der Gesundheit. Gesundheitsthemen in Sprachkursen. Praxishilfe, Essen 2007

- BKK Bundesverband (Hrsg.): BKK Gesundheitsreport. Gesundheit in Zeiten der Globalisierung, Essen 2007

- BKK Bundesverband, Gesundheit Berlin e.V. (Hrsg.): Gesund essen mit Freude. Zweisprachiges Kochbuch, 4. Auflage, Berlin 2005

- BKK Bundesverband, Gesundheit Berlin e.V. (Hrsg.): Gesund essen mit Freude. Kursmanual, Berlin 2005

- $\quad$ BKK Bundesverband, Gesundheit Berlin e.V. (Hrsg.): Gesund essen mit Freude. Leitfaden zur Unterstützung bei der Durchführung kultursensibler Koch- und Ernährungskurse, Berlin 2005

- $\quad$ BSFSJ (Hrsg.): Familien ausländischer Herkunft in Deutschland. 6. Familienbericht, Berlin/Bonn 2000

- Peters: Gesundheitliche und psychosoziale Versorgung von Migrantinnen und Migranten in Frankfurt. In: Gardemann, Müller, Remmers (Hrsg.): Migration und Gesundheit: Perspektiven für Gesundheitssysteme und öffentliches Gesundheitswesen, Berichte \& Materialien. Band 17, Düsseldorf 2000

- Prof. Schmacke: Ausgangslage und Chancen kultursensibler Prävention im Gesundheitswesen. In: Türkisch Deutsche Gesundheitsstiftung (Hrsg.) Wissenschaftliche Arbeiten über Gesundheit und Migration. Gießen 2002

- Statistisches Bundesamt: Bevölkerung mit Migrationshintergrund. Ergebnisse des Mikrozensus 2005 - Fachserie 1 Reihe 2.2 - 2005. Berlin 2007

- Stickan-Verfürth: Interkulturelle Gesundheitsförderung. In: Die BKK, Heft 4/2004

- Weiss: Macht Migration krank? Eine transdisziplinäre Analyse der Gesundheit von Migrantinnen und Migranten. Seismoverlag, 2. Auflage 2005 